\title{
Mammary-Type Myofibroblastoma: A Report of Two Cases
}

Soyeon An · Joon Seon Song Soonchan Park ${ }^{1} \cdot$ Jung Won Lee ${ }^{2}$ Kyung-Ja Cho

Department of Pathology, Asan Medical Center, University of Ulsan College of Medicine, Seoul; ${ }^{1}$ Department of Radiology, Kyung Hee University Hospital at Gangdong, Kyung Hee University School of Medicine, Seoul; ' ${ }^{2}$ epartment of Surgery, Asan Medical Center, University of Ulsan College of Medicine, Seoul, Korea

Received: December 8, 2015

Revised: March 11, 2016

Accepted: March 26, 2016

\section{Corresponding Author}

Joon Seon Song, MD, PhD

Department of Pathology, Asan Medical Center,

University of Ulsan College of Medicine,

88 Olympic-ro 43-gil, Songpa-gu, Seoul 05505,

Korea

Tel: +82-2-3010-4548

Fax: $+82-2-472-7898$

E-mail: Songjs@amc.seoul.kr
Mammary-type myofibroblastoma (MFB) is a rare, benign spindle cell neoplasm occurring along the milkline, with extension from the mid-axilla to the medial groin. It is histologically and immunohistochemically identical to MFB of the breast and is part of a spectrum of lesions that includes spindle cell lipoma and cellular angiofibroma. Recently, we experienced two cases of mammarytype MFB involving male patients aged 30 and 58 years, respectively. The tumors were located in the right scrotal sac and in the right axilla. Wide excisions were performed. Microscopically, the masses were composed of haphazardly arranged, variably sized fascicles of bland spindle cells and were admixed with mature fat tissue. The spindle cells in both cases showed immunopositivity for desmin and CD34 and negativity for smooth muscle actin. Loss of retinoblastoma $(\mathrm{RB}) / 13 \mathrm{q} 14$ loci is a characteristic genetic alteration of mammary-type MFB, and we identified loss of RB protein expression by immunohistochemical staining. We emphasize the importance of awareness of this rare neoplasm when a spindle cell neoplasm is accompanied by desmin immunopositivity. The second patient was alive without recurrence for 20 months, and the first patient had not been followed.

Key Words: Mammary-type myofibroblastoma; Milkline; 13q14
Mammary-type myofibroblastoma (MFB) is a rare, benign spindle cell neoplasm with myofibroblastic differentiation that is histologically identical to MFB of the breast and was first described by Wargotz et al. in 1987. The tumor occurs mainly in older men and postmenopausal women. The age at presentation ranges from 35 to 85 years. ${ }^{2,3}$ Common locations are along the embryonic milkline with extension from the mid-axilla to the medial groin. ${ }^{2}$ The most common presentation is a painless, slowly growing mass. ${ }^{2,4}$ The tumor is composed of bland spindle cells with myofibroblastic differentiation, prominent mast cells, fatty component, and hyalinized stroma. Recently, we experienced two cases of mammary-type MFB in the right scrotal sac of a 30-year-old man and in the right axilla of a 58-year-old man. In this report, we describe these two rare cases of mammary-type MFB.

\section{CASE REPORT}

\section{Case 1}

A 30-year-old previously healthy man presented with a soli- tary palpable mass in his right scrotal sac, which appeared 3 months earlier. He had no history of trauma or medical disease. The mass was painless, firm, and movable on physical examination. Abdominal/pelvic computed tomography (CT) showed a $5.0 \mathrm{~cm}$ mass in the right scrotal sac, separated from the adjacent testis and spermatic cord (Fig. 1A). Due the possibility of a mesenchymal tumor, excision was performed.

Grossly, the lesion was well-circumscribed and ovoid, measuring $5.0 \times 3.5 \times 2.0 \mathrm{~cm}$. The cut surface of the mass was yellowish tan, rubbery, and trabeculated, without necrosis or hemorrhage (Fig. 2A). Microscopically, the mass was composed of bland spindle cells with haphazardly arranged collagen fibers and numerous mast cells. Some areas showed edematous stroma with hypocellularity. Numerous mast cells were scattered in the stroma (Fig. 2B). There was no cellular atypia or mitotic activity. Individual adipocytes and adipose tissue were admixed with the spindle cells, which showed immunopositivity for desmin (1:200, Dako, Glostrup, Denmark) and CD34 (1:500, Immunotech, Marseille, France) (Fig. 2C, D) and negativity for smooth muscle actin 
(1:200, Dako), S-100 protein (1:100, Zymed, San Francisco, CA, USA), and human melanoma black 45 (1:50, Dako).

\section{Case 2}

A 58-year-old man presented with a slowly growing right axillary mass that had appeared 15 months earlier. A chest CT revealed a $5.5-\mathrm{cm}$ lobulated mass with infiltration of the sur- rounding soft tissue in the right axilla (Fig. 1B). Because of the possibility of metastatic carcinoma of unknown origin, a needle biopsy was performed. The diagnosis was low-grade spindle cell neoplasm with myofibroblastic differentiation. A wide excision was then performed. No evidence of recurrence was noted after 20 months of follow-up.

The mass was well-circumscribed, measuring $6.2 \times 5.2 \times 4.5$
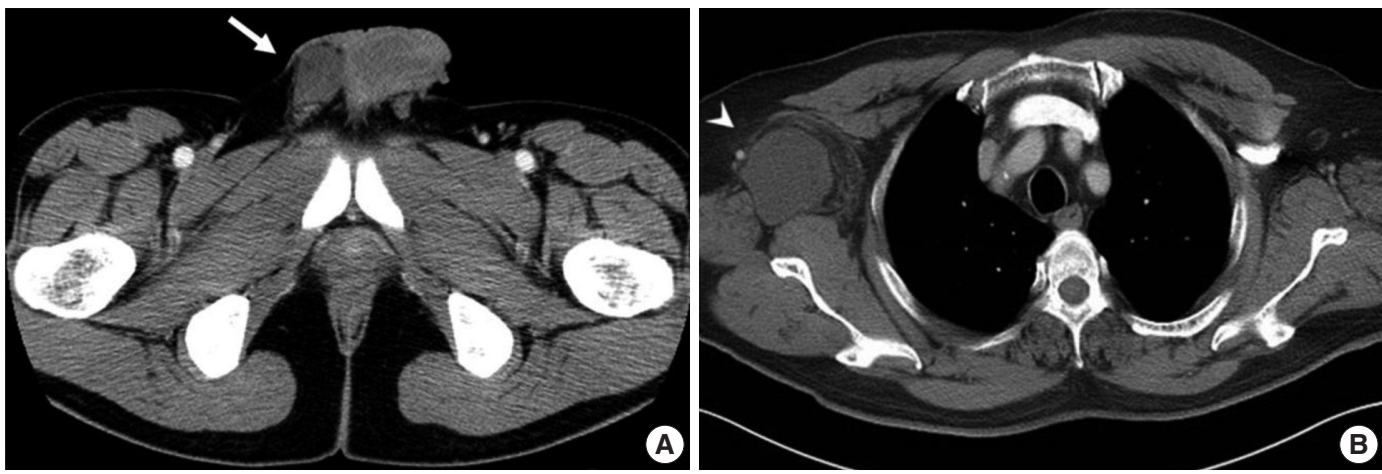

Fig. 1. Radiologic findings for the two study cases. (A) In case 1, a pelvis computed tomography (CT) shows $5.0 \mathrm{~cm}$ mass in the right scrotal sac separated from the adjacent testis and spermatic cord (arrow). (B) In case 2, a chest CT shows a $5.5 \mathrm{~cm}$ lobulated mass in the right axilla (arrowhead).
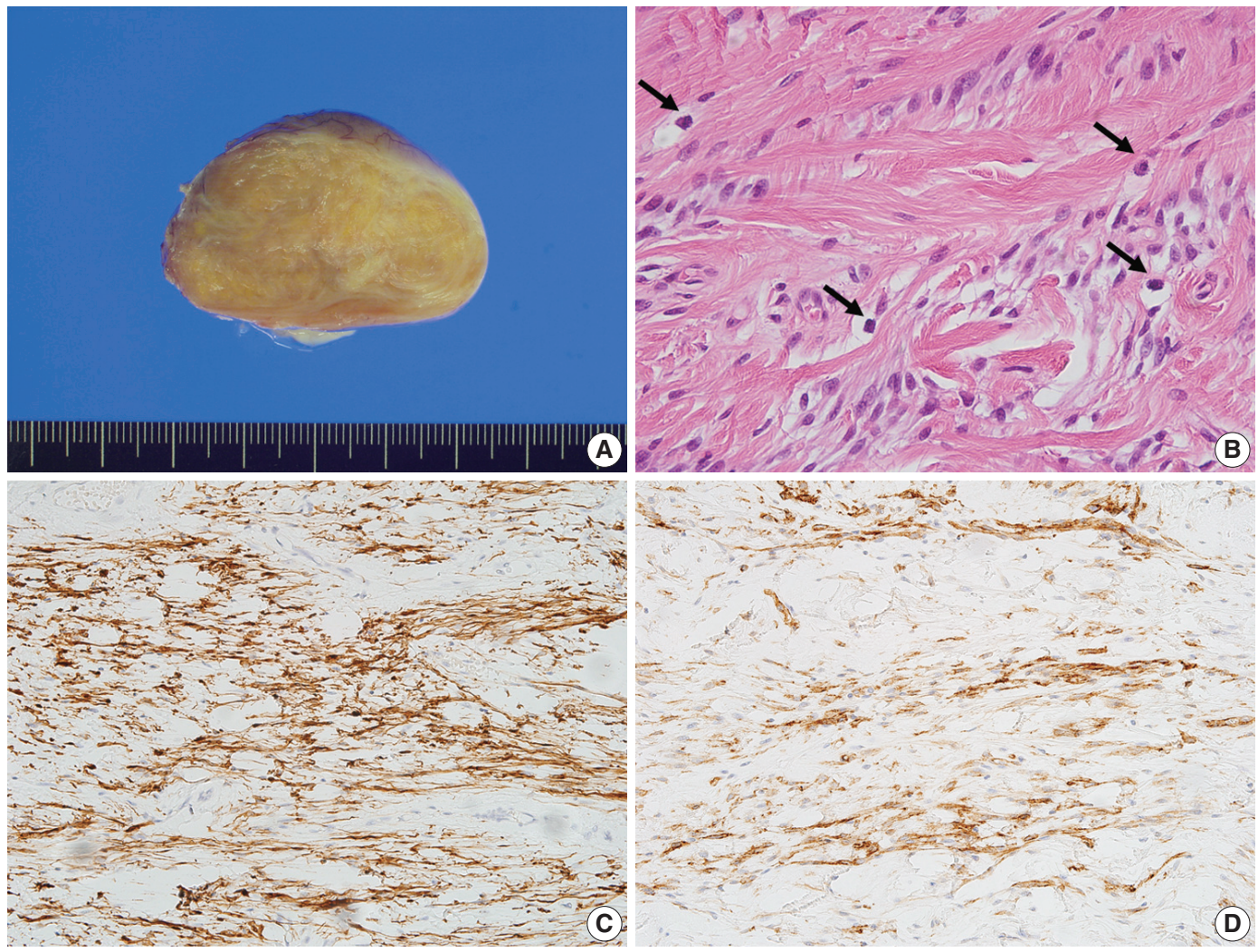

Fig. 2. Gross and microscopic findings of case 1. (A) The cut surface is yellowish tan, rubbery, and trabeculated, without necrosis or hemorrhage. (B) The mass is composed of haphazardly arranged and variably sized fascicles of bland spindle cells. Tumor cells have tapered nuclei and fine chromatin. Numerous mast cells are scattered (arrows). No mitosis is observed. The tumor cells show immunopositivity for des$\min (\mathrm{C})$ and $\mathrm{CD} 34$ (D). 

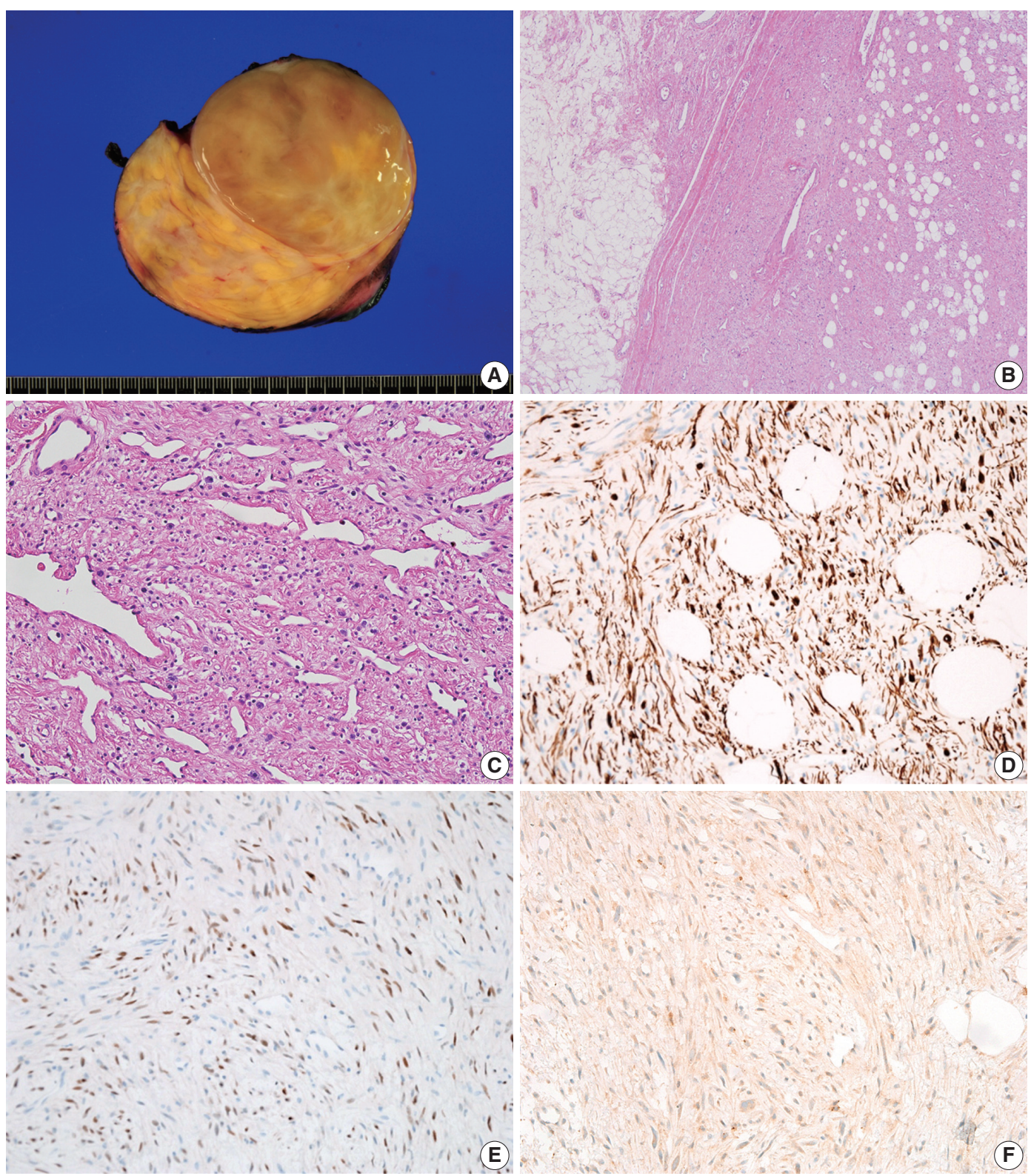

Fig. 3. Gross and microscopic findings of case 2. (A) The cut surface is yellowish tan, smooth, and glistening, and a focal myxoid change is observed. (B) The mass is well demarcated with mature adipocytes. (C) Staghorn-like blood vessels are present, with focal hyalinization. Tumor cells shows immunopositivity for desmin (D) and estrogen receptor (E). (F) Retinoblastoma immunostaining shows negativity.

$\mathrm{cm}$. The cut surface was yellowish tan, smooth, and glistening with focal myxoid change. The peripheral portion showed a more prominent fatty component (Fig. 3A). Microscopically, the mass was composed of haphazardly arranged, variably sized fascicles of bland spindle cells and numerous mast cells. The spindle cells had tapered nuclei and fine chromatin. No cellular atypia or mitotic activity was identified. Hyalinized collagen fibers were abundant in the stroma. Adipose tissue was observed in both the intra-/extra-lesional portions. Staghorn-like blood vessels were present, with focal hyalinization (Fig. 3B, C). The spindle cells showed immunopositivity for desmin, CD34, and estrogen receptor (1:100, Novo, Newcastle upon Tyne, UK) and negativity for smooth muscle actin, S-100 protein, and retinoblastoma (1:20, Dako) (Fig. 3D-F).

The Institutional Review Board (IRB) of Asan Medical Center (IRB No. 2015-1303) approved this case report.

\section{DISCUSSION}

Mammary-type MFB can occur along the embryonic milkline 
and accessory breast. Nevertheless, in many previously reported cases, the tumor did not contain any breast tissue. McMenamin and Fletcher ${ }^{2}$ described the possibility of ectopic breast in locations remote from the embryonic milkline. In addition, Millo et al. ${ }^{5}$ suggested mesenchymal CD34-positive stem cells as an explanation for the hepatic location of mammary-type MFB.

MFB cells demonstrate immunopositivity for desmin and CD $34^{2,6}$ In addition, at least focal expression of androgen, estrogen, and progesterone receptors has been reported. ${ }^{4}$ Expression of smooth muscle actin is present in one-third of MFB cases. In the two cases presented here, the tumor cells showed immunopositivity for desmin and CD34 and immunonegativity for smooth muscle actin. The differential diagnoses of mammarytype MFB include other low-grade spindle cell neoplasms such as spindle cell lipoma, cellular angiofibroma, and solitary fibrous tumors (SFTs). Desmin, CD34, and estrogen receptors are helpful markers for differential diagnosis. ${ }^{4,6}$ Recently, Howitt and Fletcher ${ }^{6}$ reported a large series of 143 cases of mammarytype MFB. They described complicated situations with mammary-type MFB, such as morphologic variation and CD34 and/ or desmin negativity. ${ }^{6}$ Spindle cell lipoma and cellular angiofibroma show overlapping histologic and immunophenotypic features with mammary-type MFB and share similar genetic findings, with loss of $13 \mathrm{q} 14$. $^{1,2,8,9}$

Spindle cell lipoma has similar histologic features to MFB such as bland spindle cells, abundant mast cells in the stroma, and admixed adipose tissue. The morphological differences between these two tumor types are subtle. The spindle cells of mammary-type MFB show a fascicular arrangement, whereas those of spindle cell lipoma show a more haphazard arrangement. CD34 expression is present in both tumors, whereas desmin immunopositivity is rarely present in spindle cell lipoma $(<2 \%) .{ }^{2}$ Maggiani et al. ${ }^{9}$ explained these different histologic features and immunoprofiles as due to stromal precursor cells with a variable capacity to differentiate into fibroblastic/lipocytic or myofibroblastic cells. Cellular angiofibroma usually involves the groin/pelvic area. ${ }^{2,10}$ Histologically, cellular angiofibroma is composed of short spindle cells arranged without any pattern and numerous small- to medium-sized thick-walled hyalinized vessels. Some tumors contain a variable amount of adipose tissue. ${ }^{2,10,11}$ Tumor cells can express CD34, but smooth muscle actin and desmin are usually negative. ${ }^{10,11}$

Mammary-type MFB, spindle cell lipoma, and cellular angiofibroma share not only histologic features, but also similar genetic alterations. The RB1 gene, as well as the FOXO1 (forkhead box protein O1, previously known as FKHR, forkhead in rhabdomyosarcoma) gene, is located in the 13q14 tumor suppressor locus, and deletion of this region, which was originally identified in spindle cell lipoma, has been reported in several cases of mammary-type MFB and cellular angiofibroma. ${ }^{9}$ Maggiani et al. ${ }^{9}$ described the loss of RB/13q14 and FKHR/13q14 loci in mammary-type MFB using fluorescence in situ hybridization (FISH) and suggested a genetic link with spindle cell lipoma, which has a characteristic loss of 13q and 16q. Hox et al. ${ }^{8}$ reported a loss of RB/13q14 loci in mammary-type MFB of the head and neck region. Magro et al. ${ }^{12}$ described a deletion of FOX01, which is located on 13q14.11, both in mammary and vaginal MFB. In that study, five of seven cases of mammary-type MFB and three of five cases of vaginal MFB showed deletion of this locus. Hameed et al. ${ }^{1}$ reported monosomy of chromosome 16 and loss of chromosome 13 in cellular angiofibroma. Flucke et al. ${ }^{10}$ described the heterozygous loss of RB1 in seven cases of cellular angiofibroma according to FISH. Magro ${ }^{13}$ suggested the term benign stromal/mesenchymal tumor with 13q14 deletion, as these three entities share the same genetic alteration, and a minority of cases of spindle cell lipoma and cellular angiofibroma showed histologic similarity to mammary-type MFB.

SFT is another possible differential diagnosis. The tumor is composed of spindle cells surrounding thick-walled, branching staghorn-like blood vessels. An admixed adipocytic component can be present. ${ }^{2,11}$ In SFT, the spindle cells show a haphazard arrangement with intertwining thin collagen fibers, whereas those in mammary-type MFB show short fascicles with broad interrupting collagen bands. Oval myoid cells with abundant eosinophilic cytoplasm, which are distinguishing features of mammary-type MFB, are not observed in SFT. SFT commonly expresses CD34 (90\%) and CD99 (70\%), especially the fibrous form. ${ }^{14}$ Although SFT shares the histological and immunohistochemical characteristics of mammary-type MFB, the cytogenetics of SFT are different from those of mammary-type MFB. The absence of $R B / 13 \mathrm{q} 14$ loss in SFTs is the key point for the differential diagnosis. ${ }^{11,15}$

We report these cases due to the rare incidence and diagnostic utility of desmin immunoreactivity. We conclude that, if spindle cell lesions with myofibroblastic differentiation and admixed adipose tissue and mast cells in unusual locations are observed, the diagnosis of mammary-type MFB should be considered as a differential diagnosis, and desmin immunopositivity could be helpful for diagnosis. 


\section{Conflicts of Interest}

No potential conflict of interest relevant to this article was reported.

\section{REFERENCES}

1. Hameed M, Clarke K, Amer HZ, Mahmet K, Aisner S. Cellular angiofibroma is genetically similar to spindle cell lipoma: a case report. Cancer Genet Cytogenet 2007; 177: 131-4.

2. McMenamin ME, Fletcher CD. Mammary-type myofibroblastoma of soft tissue: a tumor closely related to spindle cell lipoma. Am J Surg Pathol 2001; 25: 1022-9.

3. Mukonoweshuro P, McCormick F, Rachapalli V, Natale S, Smith ME. Paratesticular mammary-type myofibroblastoma. Histopathology 2007; 50: 396-7.

4. Arsenovic N, Abdulla KE, Shamim KS. Mammary-type myofibroblastoma of soft tissue. Indian J Pathol Microbiol 2011; 54: 391-3.

5. Millo NZ, Yee EU, Mortele KJ. Mammary-type myofibroblastoma of the liver: multi-modality imaging features with histopathologic correlation. Abdom Imaging 2014; 39: 482-7.

6. Howitt BE, Fletcher CD. Mammary-type myofibroblastoma: clinicopathologic characterization in a series of 143 cases. Am J Surg Pathol 2016; 40: 361-7.

7. Fletcher CD, Bridge JA, Hogendoorn P, Mertens F. WHO classifica- tion of tumours of soft tissue and bone. Lyon: IARC Press, 2013; 61-2.

8. Hox V, Vander Poorten V, Delaere PR, Hermans R, Debiec-Rychter M, Sciot R. Extramammary myofibroblastoma in the head and neck region. Head Neck 2009; 31: 1240-4.

9. Maggiani F, Debiec-Rychter M, Verbeeck G, Sciot R. Extramammary myofibroblastoma is genetically related to spindle cell lipoma. Virchows Arch 2006; 449: 244-7.

10. Flucke U, van Krieken JH, Mentzel T. Cellular angiofibroma: analysis of 25 cases emphasizing its relationship to spindle cell lipoma and mammary-type myofibroblastoma. Mod Pathol 2011; 24: 82-9.

11. Fritchie KJ, Carver P, Sun Y, et al. Solitary fibrous tumor: is there a molecular relationship with cellular angiofibroma, spindle cell lipoma, and mammary-type myofibroblastoma? Am J Clin Pathol 2012; 137: 963-70.

12. Magro G, Righi A, Casorzo L, et al. Mammary and vaginal myofibroblastomas are genetically related lesions: fluorescence in situ hybridization analysis shows deletion of 13q14 region. Hum Pathol 2012; 43: 1887-93.

13. Magro G. Chromosome 13q14 deletion in a mammary-type myofibroblastoma of the big toe: reply. Hum Pathol 2015; 46: 344-5.

14. Gengler C, Guillou L. Solitary fibrous tumour and haemangiopericytoma: evolution of a concept. Histopathology 2006; 48: 63-74.

15. Meguerditchian AN, Malik DA, Hicks DG, Kulkarni S. Solitary fibrous tumor of the breast and mammary myofibroblastoma: the same lesion? Breast J 2008; 14: 287-92. 